\title{
3
}

\section{A FORMER MAJOR PLANET OF THE SOLAR}

\section{SYSTEM}

\author{
T. C. VAN FLANDERN
}

Dynamical calculations by Ovenden, indicating the former existence of a 90-Earth-mass planet in the asteroid belt, have now been supported by a study of orbital element distributions of very-long-period comets. The indicated epoch for disintegration of the planet is just $5 \times 10^{6}$ years ago.

\section{INTRODUCTION}

The asteroid belt has been suspected of being the by-product of a former planet since its discovery. This paper summarizes evidence supporting this asteroidal-planet theory, and uses dynamical methods to demonstrate that comets probably also originated in the breakup of such a planet at a recent epoch.

The Canadian dymamical astronomer M. W. Ovenden has already presented the results of calculations based upon the so-called "principle of least interaction action" (Ovenden 1972). In essence, the principle predicts a Bode's-1aw-type arrangement of the solar system planets; and, similar to the original Titius-Bode law, an additional planet in the present location of the asteroid bel hetween Mars and Jupiter, at about $2.8 \mathrm{AU}$ from the Sun, is to be expected.

Ovenden's principle is specific enough, however, to enable the mass and approximate epoch of dissipation of the missing planet to be determined from the rates of evolution of the orbits of the remaining planets, which are still undergoing an adjustment due to the disappearance of the planet in question. The former asteroidal planet would have had a mass of $90 \pm 5$ Earth-masses (about Saturn's size), and would have been present until an estimated $16 \times 10^{6}$ years ago. Inasmuch as less than $10^{-3}$ Earth-masses remains today in the form of asteroids, and since no core or single large fragment remains, most of the mass of the planet apparently disappeared from the solar system, either by escape or by collision with the Sun and planets.

\section{METEORITIC EVIDENCE}

Ovenden (1973) has also cited evidence for a recent break-up event from the chondritic meteorites. Although typical meteorite ages range from hundreds of millions to billions of years, the carbonaceous chondrites seem nearly all to have cosmic ray exposure ages of less than ahout $22 \times 10^{6}$ years. Given the many sources of uncertainty in this dating method, this particular class of meteorites is very likely associated with the asteroidal planet break-up. Indeed it is possible that most "young" meteorites came from this event, with their 


\section{VAN FLANDERN}

exposure ages falsified (increased) by radioactive processes associated with whatever caused the break-up.

\section{EVIDENCE FROM COMETS}

New evidence is now available from very-long-period comets, which apparently also originated from the break-up. It was previously known (Everhart 1972) that the distribution of orbital elements of short-period comets could be explained by the action of planetary perturbations on long-period comets; hence it is only necessary to understand the origin of the latter to explain hoth types. One of the first predictions of an energetic planetary hreak-up is that there should exist some objects ejected with velocities just below that of escape, which would now be making their first return to perihelion since ejection. Such a class of comets has been known to exist since the work of Oort (1950), recently confirmed by Marsden and Sekanina (1973). These comets all have similar periods (on the order of $10^{7}$ years) and similar dimensions (aphelia at about 60,000 a.v.) -- characteristics which could not have survived even a single previous perihelion passage. The precise periods, while somewhat uncertain, are close to $6 \times 10^{6}$ years.

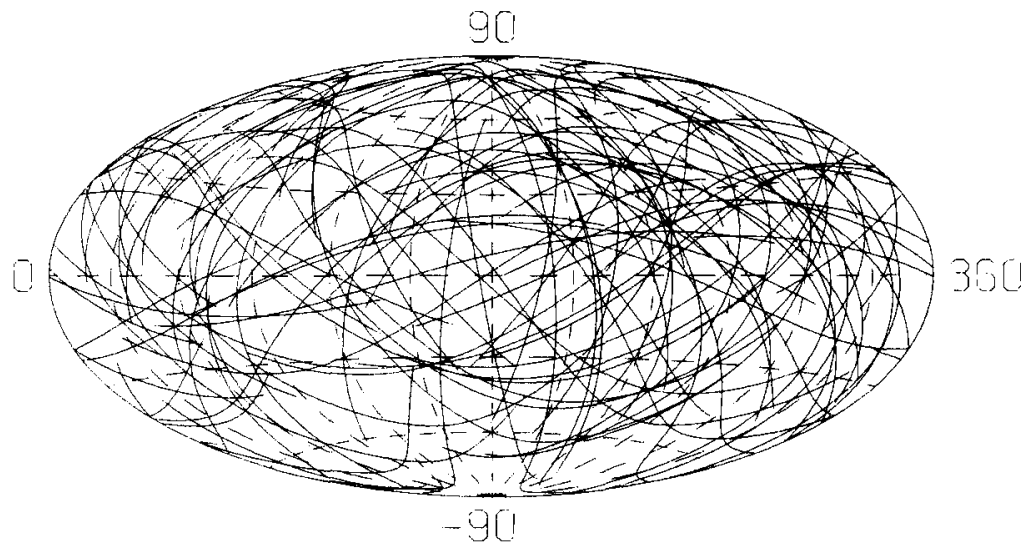

Figure 1. 60 very-long-period comet orbits on heliocentric celestial sphere at present epoch, in ecliptic coordinates centered at longitude $180^{\circ}$.

In the comet catalog of Marsden (1975) there are about 60 of these very-1ongperiod comets with well-determined orbits. A very important characteristic of these orbits, predicted by the Ovenden planet model, is that, to the extent that they have not been perturbed, they should all intersect on the celestial sphere (as viewed from the Sun), at the same point - the point of the break-up. Figure 1 shows a plot of the portion of these 60 orbits within the solar system during their most recent apparition. Although the orbits scatter all over the sky, there is a tendency to cluster near $249^{\circ}$ ecliptic longitude. The clustering is quite statistically significant; at the center of the cluster, four orbits intersect within 0.01 square degrees. (Since each heliocentric orbit is a portion of a great circle, a symmetric clustering may appear at the diametrically opposite point on the sphere).

If most of these comets have just completed their first revolution since the break-up, then the principal sources of perturbations on their orbits would be from the tidal effects of the galactic field, with a randomizing effect due to stellar encounters superimposed. The galactic perturbations can be almost 


\section{A FORMER MAJOR PLANET?}

completely eliminated by numerical integration of the equations of motion backwards in time to the previous perihelion passage. If this is done, and the orbits are traced on the heliocentric celestial sphere as they would have appeared about $6 \times 10^{6}$ years ago, the result is shown in Figure 2. The clustering in Figure 1 has now become quite pronounced, and has moved to ecliptic longitude $258^{\circ}$ and quite close to the ecliptic plane. The scatter in the remaining orbits is consistent with expectations due to random stellar encounters, which can be estimated at \pm 2.1 radians in $6 \times 10^{6}$ years, based on the density of stars in the solar neighborhood. If the integrations are pressed further back in time, the galactic perturbations become so large for orbits with periods of $10^{7}$ years that sensitivity to the initial conditions is lost, and the integrations are not reversible. If comets with such large periods existed, their orbits would have been found to be aligned with the galactic center at the discovery epoch.

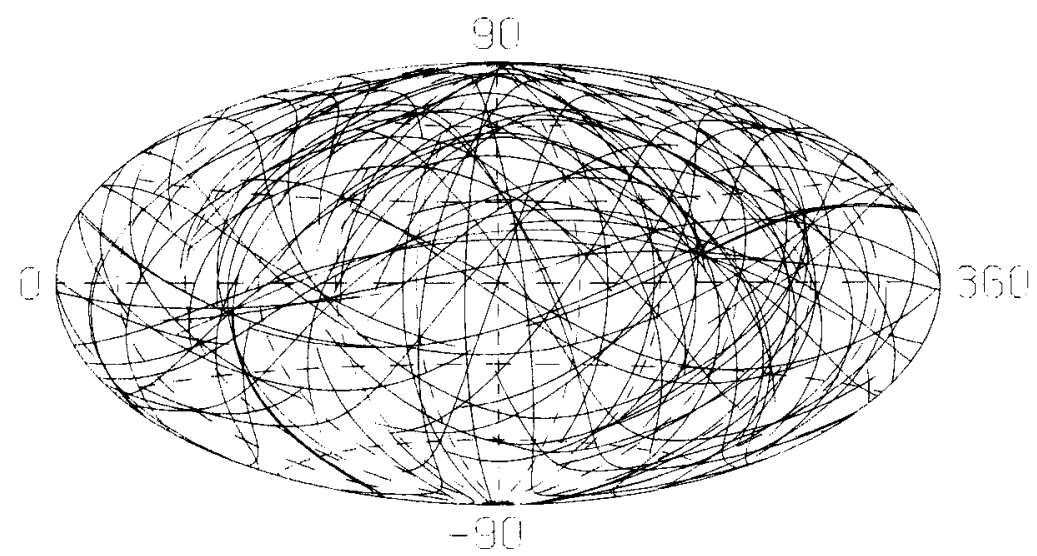

Figure 2. Same as Figure 1, but for the epoch 5,800,000 years ago.

Similar evidence is present in each orbital element for the long-period comets. From the orbital parameters of the original planet, certain predictions can be made about the expected distributions of comet elements. For example at the break-up, more particles will have initial velocities nearly perpendicular to the solar radius vector (two dimensions) then nearly parallel to it. As a consequence, for particles moving very close to escape velocity (and therefore in nearly-parabolic orbits), it can be derived that the Sun will eject about twice as many particles toward one hemisphere of the sky as toward the other. In reality, twice as many perihelia lie in the hemisphere centered on longitude $258^{\circ}$ as in the other (see Figure 3), in excellent agreement with the prediction. This characteristic is virtually immune to galactic and stellar perturbations, and is very difficult to explain with any other proposed theory of cometary origin.

Inspection of the distribution of orbital inclinations and perihelion distances of these same 60 comets near the epoch $6 \times 10^{6}$ years ago yields the information that the angular momentum and solar distance of the source body are consistent with those of a planet in direct motion about the sun close to the ecliptic, at a distance which could not differ from 2.8 astronomical units by much more than a factor of two.

OORT CLOUD MODEL

It is usually thought that comets circle the Sun at distances of near 60,000 


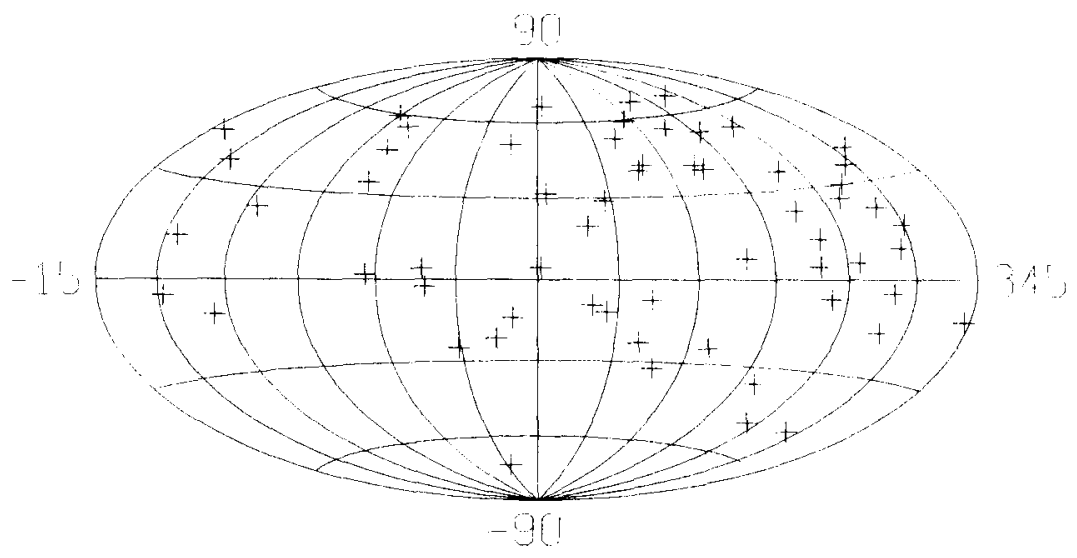

Figure 3. Perihelion directions of 60 very-long-period comets in ecliptic coordinates centered at longitude $165^{\circ}$.

a.u., and are occasionally perturbed by stellar encounters in such a way as to cause them to pass near the Sun. This model not only fails to predict the correct distribution of perihelion direction and inclination and the orbital plane clustering; but it makes a specific prediction about the perihelion distance distribution which is contradicted by the observations. For near parabolic orbits the perihelion distance $q$ should be randomized for comets which are perturbed by stellar encounters as in the Oort cloud model. Therefore, we would expect there to be approximately the same number of comets with q values between 0.5 and 1.0 as the number with $q$ values between 1.0 and $1.5 \mathrm{a} . \mathrm{u}$. Yet the actual number of comets is 9 for the first range and 16 for the second. When the effect of discovery probabilities, which favors the first of these two ranges, is included, the probability is less than 0.03 that this bias occurred hy chance, which argues against the Oort cloud model. The planetary break-up model predicts increasing numbers of comets in each range out to the asteroid belt.

\section{CONCLUS ION}

Ovenden's calculations demonstrated the existence of a former massive planet in the asteroid belt until an astronomically-recent epoch. These calculations are now strikingly supported by characteristics of very-long period comet orbits, which must have originated in the break-up of the planet. Although existing evidence provides few clues as to how such an event could have happened, the independent evidence from dynamical calculations, meteoritic dating, and comet orbit characteristics (especially the clusterings of periods, orbital planes, and perihelion directions for comets) are convincing that the solar system did indeed have a large tenth planet until an epoch so recent that primates were already present on the Earth, and that the break-up of that planet provided the origin of comets and asteroids.

Although the information presented in this short article is insufficient to document the validity of the conclusions drawn, and must inevitably leave a host of questions unanswered in the mind of any interested reader, the more extensive and detailed documentation is in preparation, and will be published as rapidly as possible in the appropriate journal. 


\section{A FORMER MAIOR PLANET?}

\section{REFERENCES}

Everhart, E. 1972, Astroph. Letters, 10, 131.

Marsden, B. G., and Sekanina, Z. 1973, Astron. J., 78, 1118.

Marsden, B. G. 1975, Catalogue of Cometary Orbits, Smithsonian Astrophysical Observatory, Cambridge, Mass.

Dort, J. H. 1950, Bul1. Astron. Inst. Neth., 11, 91.

Ovenden, M. W. 1973, in Recent Advances in Dynamical Astronomy, B. D. Tapley and $V$. Szebehely, eds.

Ovenden, M. W. 1972, Nature, 239, 508.

\section{DISCUSSION}

ANDERS: Before going on to more fundamental arguments, let me respond to your suggestion that the sinuous rilles on Mars and the Moon were caused by impacts of large, icy fragments of the hypothetical disrupted planet. The rilles on Mars have tributaries and other features of river beds, and undoubtedly were carved by flowing water. But from their morphology, it seems that the water had an internal, not an impact origin. The rilles on the Moon have no tributaries and apparently represent collapsed lava tubes. One such rille, Rima Hadley, was visited during the Apollo 15 mission, and neither field observations by the astronauts nor laboratory study of the returned rocks, of age $3.4 \times 10^{9}$ yr, gave any indication for the occurrence of water, past or present.

One major objection to your idea is the impossibility of breaking up a planet of 93 Earth masses. A collision with another body will not do it, as you yourself have admitted. Öpik some years ago discussed other mechanisms, such as phase transformations, for disrupting planets, and found that they wouldn't work even for large asteroids, let alone Saturn-sized bodies. The total energy required is so huge that only a nuclear explosion can meet it, but such an explosion 6 Myr ago would have left large amounts of long-lived radioactivities, such as $\mathrm{Be}^{10}$ and $\mathrm{Mn}^{53}$, on the Earth, Moon, and meteorites, Moreover, a nuclear explosion, like an impact, would raise the internal energy of the ejecta along with the kinetic energy, so that material accelerated to more than a couple of $\mathrm{km} / \mathrm{sec}$ would be melted or vaporized. (The absence of lunar rocks from the Earth's meteorite collections is an illustration of this fact).

A second objection is that raised by Prof. Gold. Disruption of a Saturnsized body in the asteroid belt 6 Myr ago would have led to a vast increase in meteorite $f l u x$ and hence cratering rates on the Earth, Moon, and Mars. Such increases in meteorite flux have been looked for, and the only hint is an apparent slight increase in the flux $500 \mathrm{Myr}$ ago, as inferred by Neukum from lunar crater counts. [He attributes it to the disruption of the L-chondrite parent body 520 Myr ago (Anders 1964, 1965; Heymann 1967), a very modest event involving a medium-sized asteroid]. Similarly, the cosmic-ray exposure age distribution of meteorites shows no evidence of a major event 6 Myr ago. It is a continuum, extending to $10^{9}$ yr for irons and or -50 Myr for stones, and although there are occasional peaks superimposed on the continuum, none are at 6 Myr.

Third, most of the larger asteroids are nearly spherical, and so is 1.566 Icarus, a l-km object thought by many to be an extinct cometary nucleus. Fragments from a breakup should be angular, not spherical.

Fourth, chondrites contain enough $K, U$, and $T h$ to cause melting and differentiation in bodies of lunar size or larger. Thus a Saturn-sized planet would be compositionally layered. On breakup, these layers would have to be mixed back in the original proportions, on a scale of $0.1 \mathrm{~cm}$ (the smallest cometary meteors that have spectra corresponding to chondritic composition).

In summary, there is no evidence favoring the existence of an asteroidal planet except the slight asymmetry of cometary perihelia. In view of the com- 
plete absence of all further evidence, and the need to appeal to supernatural processes for disruption of a planet, it would seem more profitable to look for other, more prosaic, explanations of the asymmetry.

VAN FLANDERN: You have raised a number of issues, none of which bear directly on the data or the interpretation $I$ have presented, but which rather speak to the necessarily speculative details of the method and circumstances surrounding the disruption of a large planet.

Quite obviously the Ovenden hypothesis is in sharp conflict with the existing interpretations of most comet, asteroid, and meteoritic data. This is irrelevant. The important question is whether there is any conflict with the data, rather than with existing interpretations of the data, This is, I think, a very important point.

For example, it is widely believed that chondritic material condensed directly from the solar nebula. The very young exposure ages are "explained" by invoking recent break-ups of slightly larger bodies. But if meteoritic material was inside a large planet until recently, all cosmic ray exposure ages might be falsified, either by ongoing nuclear processes within the planet, or during the energetic break-up event itself. As with the popular interpretation, this is a speculation. But we must not be lured into using familiarity as a criterion for judging hypotheses.

Regarding specific points, P. M. Muller is at least one authority whose recent publications have concluded that the issue of water on the Moon is not yet closed. The collapsed lava tube explanation is becoming increasingly difficult to believe, and some sinuous rilles do have tributaries.

About break-up mechanisms, I submit that if we were not faced with incontrovertible evidence that stars explode, modern astrophysics would convincingly conclude that that was impossible also. Ovenden has pointed out that the range of stable conditions for planets is so narrow that it is more difficult to understand how to prevent them from breaking up.

On meteorite fluxes, a small increase in the flux at $6 \times 10^{6}$ years ago would surely have gone unnoticed. The hypothetical break-up would provide a natural explanation for things like tektites, and the excess of small impact craters on Mars.

Nothing in this hypothesis prevents Icarus from being an extinct cometary nucleus. And we know so little about the solid interiors of Jupiter and Saturnsized bodies that I am surprised by your compositional argument.

Finally, if I have left the misimpression that my analysis rests on nothing more than a slight asymmetry of comet perihelia, I would like to correct it. The distributions of every orbital element of the very-long period comets is consistent with a break-up origin, and several of the distributions have high statistical significance. Perhaps there is another explanation, though I doubt it; but to ignore the clear message in the data surely cannot be the way to advance knowledge.

MARSDEN: I do not believe that one can place much significance on possible nonrandom effects that appear in your Figures 1 and 3 -- and even in your more crucial Figure 2. It seems to me that your model for calculating perturbations will automatically cause the orbit intersections to tend to line up in the directions of the center and anticenter of the Galaxy. This focusing effect must be even more pronounced if you assumed that the periods of the comets were, say, 20 million years, a compromise between the planet-disintegration date given by ovenden and what one might deduce from meteorite cosmic-ray exposure ages. of course, we know that any comets with periods of 20 million years would be seriously influenced by individual nearby stars, but the same must to some extent have been true of the known "first-pass" comets with half-periods of 2-3 million years since their aphelion passages. Since this influence must basically 
be due to the action of a relatively small number of individual stars over a rather short interval of time, there is absolutely no reason why such action should be random, so the possible existence of minor systematic effects can certainly not be regarded as a condemnation of the oort cloud concept. Given a sample of only 60 presumed first-pass comets, I am not unduly surprised that more than twice as many of them should have perihelion directions in one hemisphere as in the other, although my own examination of the orbits of what $I$ believe might be first-pass comets suggests an asymmetry that is considerably smaller than this.

VAN FLANDERN: In recent correspondence between us you have, in the manner of any conscientious scientist who is skeptical, attacked my strongest argument first, namely the perihelion direction clustering in Figure 3 . This is an especially important argument because it is not affected significantly by either galactic or stellar perturbations. After several exchanges we agreed that my sample had $65 \%$ of perihelia in one hemisphere, yours had $60 \%$, and an independent and larger sample of long-period comets with elliptical orbits had a $64 \%$ bias, all statistically significant. Retaining the option to return to this argument later, you then questioned the significance of the clustering effects in Figures 1 and 2. Your remark about individual stellar perturbations being capable of producing these effects turns out to be incorrect, since comets presumably do not. station themselves in preferred locations with respect to the track of a passing star; and the results to be expected from stellar perturbations have now been calculated. Six million years is the mean age given by the observationallydetermined periods of these comets, as well as by the clustering effects in the backwards integrations. The galactic aligning influence you spoke of is important, but not by any means dominant after six million years; and a separation from the intrinsic clustering (which just happens to lie close to the plane of the ecliptic and at the longitude expected from Figure 3) can be accomplished. You have not yet commented on my statistical argument that the "Oort cloud" model predicts something about the perihelion distance distribution which is in conflict with observations, or on the other element distributions, which you have seen in preprint form.

DELSEMME: The energy difficulties of breaking up a planet of 90 earth masses, properly described by Anders, have been discussed in detail by $\mathrm{W}$. MCD. Napier and $R$. J. Dodd (Nature, 1973242,250 ) in their response to Ovenden's attempt. In particular, masses less than 24,000 earth masses are too small to become hot enough for nuclear reactions, and any conceivable non-nuclear explosion would lack the energy needed by several orders of magnitude.

Another direct observational difficulty met by van Flandern's hypothesis is the existence of the sharp and narrow peak in the binding energy of the observed flux of "new" comets (this book, p. 460, Fig. 3). It does not seem to be easy to explain by a recent planet break-up, whereas it is a natural consequence of stellar perturbations on the margin of oort's cloud. 